Document downloaded from:

http://hdl.handle.net/10251/132417

This paper must be cited as:

Benalcazar-Parra, C.; Garcia-Casado, J.; Ye Lin, Y.; Alberola-Rubio, J.; López-Corral, A.; Perales Marin, AJ.; Prats-Boluda, G. (2019). New electrohysterogram-based estimators of intrauterine pressure signal, tonus and contraction peak for non-invasive labor monitoring. Physiological Measurement. 40(8):1-12. https://doi.org/10.1088/1361-6579/ab37db

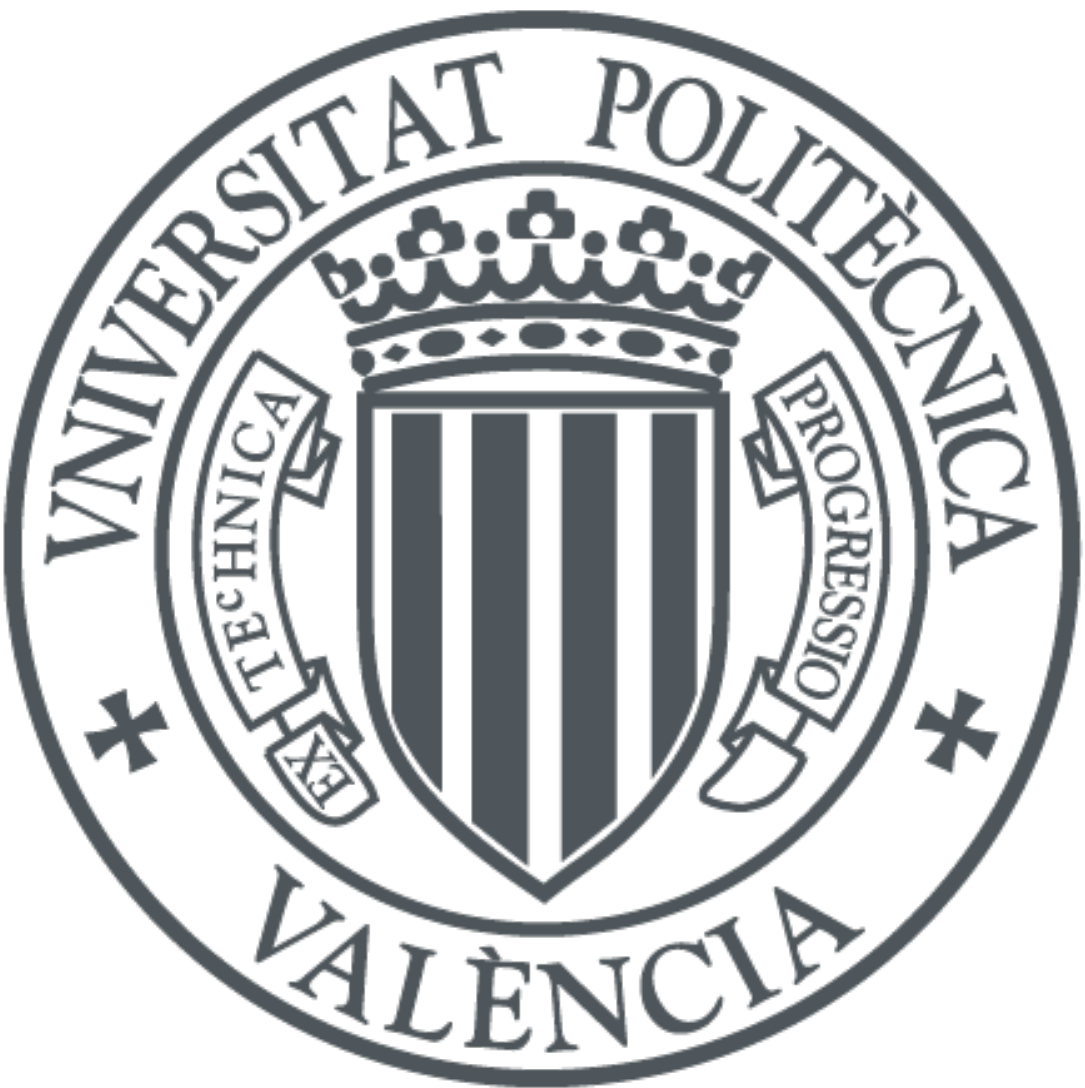

The final publication is available at

https://doi.org/10.1088/1361-6579/ab37db

Copyright IOP Publishing

Additional Information 
Title:

\section{New electrohysterogram-based estimators of intrauterine pressure signal, tonus and contraction peak for non- invasive labor monitoring.}

Running heads:

New EHG-based IUP estimators

Authors:

Carlos Benalcazar-Parra MSc ${ }^{\mathrm{a}}$, Javier Garcia-Casado $\mathrm{PhD}^{\mathrm{a}}$, Yiyao Ye-Lin Phd ${ }^{\mathrm{a}}$, Jose Alberola-Rubio $\mathrm{PhD}^{\mathrm{b}}$, Ángel Lopez MSc ${ }^{\mathrm{b}}$, Alfredo Perales-Marin $\mathrm{MD}^{\mathrm{b}, \mathrm{c}}$ and Gema Prats-Boluda $\mathrm{PhD}^{\mathrm{a}}$

\section{Affiliations}

a. Centro de Investigación e Innovación en Bioingeniería, Universitat Politècnica de València, Edif. 8B, Camino de Vera SN, 46022 Valencia, Spain. \{carbepar, yiye, jgarciac,gprats\}@ci2b.upv.es

b. Servicio de Obstetricia y Ginecología, Hospital Universitario y Politécnico La Fe de Valencia, Valencia, Spain.palberola.rubio@gmail.com,alopezlola@gmail.com, perales_alf@gva.es

c. Facultad de Medicina, Universidad de Valencia, Av Blasco Ibañez 15, 46010 Valencia, España. perales_alf@gva.es

*Corresponding author:

Full name: Javier Garcia-Casado

E-mail address: jgarciac@ci2b.upv.es

Full postal address: Centro de Investigación e Innovación en Bioingeniería, Universitat Politècnica de València, Edif. 8B, Camino de Vera SN, 46022 Valencia, Spain

Telephone: +34 963877007 ext. 76027

\section{Conflicts of Interest statement}

In accordance with my ethical obligation as a researcher, I declare that this research has received funding from the Spanish Ministry of Economy and Competitiveness and the European Regional Development Fund (DPI2015-68397-R), the Generalitat Valenciana (GV/2018/104) and UPV-IIS La Fe (UPV_FE-2018-C03). 


\begin{abstract}
Background: Uterine activity monitoring is an essential part of managing the progress of pregnancy and labor. Although intrauterine pressure (IUP) is the only reliable method of estimating uterine mechanical activity, it is highly invasive. Since there is a direct relationship between the electrical and mechanical activity of uterine cells, surface electrohysterography (EHG) has become a noninvasive monitoring alternative. The Teager energy operator of the EHG signal has been used for IUP continuous pressure estimation, although its accuracy could be improved. Objective: We aimed to develop new optimized IUP estimation models for clinical application. Approach: We first considered enhancing the optimal estimation of IUP clinical features (maximum pressure and tonus) rather than optimizing the signal only (continuous pressure). An adaptive algorithm was also developed to deal with inter-patient variability. For each optimizing signal feature (continuous pressure, maximum pressure and tonus), individual (single patient), global (full database) and adaptive models were built to estimate the recorded IUP signal. The results were evaluated by computing the root mean square errors: continuous pressure error $(\mathrm{CPe})$, maximum pressure error (MPe) and tonus error (TOe). Main results: The continuous pressure global model yielded IUP estimates with $\mathrm{CPe}=14.61 \mathrm{mmHg}, \mathrm{MPe}=29.17 \mathrm{mmHg}$ and $\mathrm{Toe}=7.8 \mathrm{mmHg}$. The adaptive models significantly reduced errors to $\mathrm{CPe}=11.88, \mathrm{MPe}=16.02$ and Toe $=5.61 \mathrm{mmHg}$. The EHG-based IUP estimates outperformed those from traditional tocographic recordings, which had significantly higher errors $(\mathrm{CPe}=21.93, \mathrm{MPe}=26.97$, and $\mathrm{TOe}=13.96)$. Significance: Our results show that adaptive models yield better IUP estimates than the traditional approaches and provide the best balance of the different errors computed for a better assessment of the labor progress and maternal and fetal wellbeing.
\end{abstract}

\title{
Keywords:
}

Intrauterine pressure; Uterine activity; Surface Electromyography; Electrohysterography; Teager energy 


\section{Introduction}

Monitoring uterine contractions is essential during pregnancy and labor to obtain information on time-to-delivery and on maternal and fetal well-being. Uterine activity (UA) is typically evaluated from records based on pressure measurements. For accurate information on UA the intrauterine pressure (IUP) is considered to be the gold standard. This technique gives quantitative UA information, such as baseline tone (resting pressure between contractions), duration (time between onset and offset of contraction), intensity (pressure rise above baseline tone caused by contractions), frequency (number of contractions in a 10-minute period) and relaxation time (interval between the end of one and onset of the next contraction) (Bakker et al 2007).

During labor uterine contractions increase progressively in peak amplitude from approximately 25 $\mathrm{mmHg}$ in the latent phase to $50 \mathrm{mmHg}$ in the active phase, in frequency from 3 to 5 contractions every 10 minutes and in baseline tone from 8 to $12 \mathrm{mmHg}$ (Cunningham et al 2010). At the end of the active phase UA rises further to an intensity of between 80 to $100 \mathrm{mmHg}$ at a frequency of 5-6 contractions every 10 minutes (Gilbert 2011), while the basal tone can reach a resting value of $16 \pm 7$ mmHg (Dowdle 2003). Obstetricians usually monitor contraction intensity to obtain information on whether uterine pressure is high enough to expel the fetus or whether medical intervention is advisable. UA monitoring is also important since abnormalities such as tachysystole (contractions in excess of five in $10 \mathrm{~min}$ ) or hypertonus (tone over $25 \mathrm{mmHg}$ (Freeman et al 2012)) could affect fetal health (Bakker et al 2007, Cunningham et al 2010). UA may also be assessed in Montevideo units (MU) to determine whether labor is progressing satisfactorily (Caldeyro-Barcia et al 1957). UA monitoring can also interpret fetal heart rate variations to assess fetal well-being. However, IUP is not commonly used in clinical practice since introducing a pressure catheter into the uterine cavity is considered invasive after membrane rupture and is associated with an increased risk of bacterial infection, maternal fever, and the need for a caesarean delivery (Harper et al 2013).

Due to its non-invasiveness, UA is often measured by a tocodynamometer (TOCO) (Euliano et al 2013) through a pressure sensor on the abdominal surface that responds to changes in abdominal contours and indirectly measures uterine contractions (Schlembach et al 2009). However this technique often experiences loss of contractions (Euliano et al 2013), can cause discomfort to the

patient and the measurement is highly dependent on sensor position and movements. Moreover, the technique cannot accurately measure the intensity, tone or duration of the contractions (Bakker et al 2007) but can only register their frequency.

Electrohysterography (EHG) has recently emerged as an alternative method of noninvasive UA monitoring (Euliano et al 2013, Alberola-Rubio et al 2013a, Ye-Lin et al 2013, Schlembach et al 
2009). EHG registers the myoelectrical activity of the uterine cells sensed by electrodes on the abdominal surface and has been shown to be more sensitive than TOCO in detecting uterine contractions (Euliano et al 2013, Alberola-Rubio et al 2013b, Benalcazar-Parra et al 2017a). It is also valuable in other applications such as: predicting preterm labor (Fergus et al 2013, Maner et al 2003, Lucovnik et al 2011, Mischi et al 2018, Hassan et al 2013), classifying labor and non-labor contractions (Hassan et al 2010), predicting type of delivery (spontaneous vs induced) (AlberolaRubio et al 2017), and characterizing uterine myoelectrical response to labor induction drugs (Benalcazar-Parra et al 2017a), although few studies have focused on this method of obtaining IUP estimates (Jezewski et al 2005, Skowronski et al 2006, Rabotti et al 2008, Rooijakkers et al 2014).

(Jezewski et al 2005) proposed the root mean square (RMS) value of the EHG to estimate the contraction pattern, compared it with TOCO signals and found that the estimated contraction pattern showed a high correlation with TOCO. However, they did not reproduce the IUP signal and their study focused on the analysis and comparison of consistent contractions.

(Skowronski et al 2006) used a Wiener filter prediction to obtain IUP estimates from rectified EHG signals. The results showed that it is feasible to noninvasively estimate IUP from EHG recordings. The method required a 10-minute initialization period, during which the IUP signal was measured (desired signal in the Wiener filter algorithm) to obtain the Wiener filter coefficients, after which the model could be applied to the EHG signal. However, this method is unsuitable for pregnancy and long-term observations, since the membranes have to be ruptured to obtain IUP.

(Rabotti et al 2008) estimated IUP by working out the unnormalized first statistical moment of the frequency spectrum (UNFM) and then improved the estimation accuracy on a second-order polynomial model. The model's coefficients were calculated for each contraction and patient, while the constant term was set to zero as they removed the baseline tone for the analysis. Then the coefficients of a global model were obtained by computing the median values of the coefficients obtained in the contraction segments from all the subjects. The fact that they remove the baseline tone limits this method as regards the correct diagnosis of hypertonus.

(Rooijakkers et al 2014) proposed a low-complexity intrauterine pressure estimate by computing the Teager energy (TE) operator of the EHG signal. TE yielded a lower root mean square error (RMSe) than the above-mentioned methods using the same data. Although good correlation coefficients were reported between the estimated signals and IUP, the mean RMSe of the estimates was greater than $14 \mathrm{mmHg}$ for all the methods compared in (Rooijakkers et al 2014). Despite the clear advantages of the proposed TE-based method over the others, its main limitation is inter-patient variability, i.e large inter-patient variations in signal amplitude are not accompanied by significant differences in the intensity of IUP contractions, giving rise to higher RMSe values. 
This variability could be due to differences in body constitution or obstetrical factors and it may be possible to improve IUP estimates by compensating for inter-patient EHG differences. To the authors' knowledge this has not been addressed so far. (Yang et al 2017) recently proposed estimating intrauterine pressure using Hilbert phase slips and statistical methods to reduce motion artifacts. Their method divides the EHG signal into segments by Hilbert phase slides. The standard deviation is then used to estimate the IUP of each EHG signal segment, followed by a median filter to eliminate motion artifacts. This approach correlates well with IUP signals and accurately detects contractions. However, the outcome is not an estimate of the IUP in $\mathrm{mmHg}$ units, but only reproduces the IUP waveform.

Furthermore, the coefficients of the models described in the literature were calculated by the least squares method, which optimizes the mean square error of the difference between each estimated sample and the corresponding IUP sample (continuous pressure optimizing). However, from a clinical point of view, a good estimate of IUP features such as contraction peak amplitude and/or tonus would be of greater interest. In this context we therefore aimed to design IUP estimation models, computing the EHG signal TE by means of different types of optimizing signal features: continuous pressure, maximum pressure and tonus. Individual and global models were built for each signal feature and we then tackled the inter-patient variability problem by developing adaptive algorithms to improve the accuracy of the IUP estimates derived from the global models.

\section{Materials and methods}

\subsection{Signal acquisition}

Simultaneous IUP and EHG signals were recorded from twenty-two women in the active phase of labor at the Hospital Universitario y Politécnico de La Fe, in Valencia, Spain. The study adhered to the Helsinki Declaration and was approved by the local medical ethical board. All the women were informed of the nature of the study, the recording protocol and provided written informed consent. Table 1 shows the obstetrical characteristics of the subjects.

The duration of the recording ranged from 1 to 4 hours. The skin was carefully prepared using exfoliating gel (Nuprep, Weaver and Company, USA) to reduce skin-electrode impedance. Four monopolar disposable $\mathrm{Ag} / \mathrm{AgCl}$ electrodes (3M red dot 2560) were then placed on the patient's abdomen (see Figure 1): 2 on each side of the median line over the navel at $8 \mathrm{~cm}$ inter-electrode distance (M1 and M2), 1 reference electrode on the right hip and 1 ground electrode on the left hip. The electrodes were connected to an ad hoc biosignal amplifier to amplify and filter EHG signals between $[0.1,150] \mathrm{Hz}$. The signals were digitalized at a sampling frequency of $500 \mathrm{~Hz}$. The IUP signal was simultaneously recorded by means of a pressure catheter (Intran Plus IUP-450, Utah 
Medical products Inc, USA) in the uterine cavity connected to a Corometrics250cx series monitor (GE HealthCare, General Electric Company, USA). The maternal-fetal monitor transmitted the data to a PC via serial port at a sampling rate of $4 \mathrm{~Hz}$. Both the EHG and IUP signals were stored for subsequent analysis. Although there is no additional clinical advantage to recording TOCO when the IUP recording is available, TOCO was measured at the same time in the last 7 sessions for an additional comparison of EHG-based IUP estimates with TOCO. For this specific analysis, TOCO records were preprocessed: zero readings were linearly interpolated with neighboring data and an additional segmentation to IUP, EHG and TOCO signals was performed to remove the TOCO intervals with loss of sensor contact. The same intervals were considered for the computation of estimates errors in both TOCO and EHG approaches and compared.

\section{$2.2 \quad$ Signal processing}

To eliminate low-and high-frequency interference and noise, EHG signals were digitally bandpass filtered between $0.2-1 \mathrm{~Hz}$ and then down-sampled at $20 \mathrm{~Hz}$ to reduce the data and computational cost, giving rise to preprocessed M1P and M2P signals, after which a bipolar EHG signal (M1P$\mathrm{M} 2 \mathrm{P}$ ) was computed to reduce common mode interference. The motion artifact segments were visually identified by experts and excluded from the study.

Teager energy was computed to estimate IUP waveforms; this consisted of calculating the instantaneous energy, which is directly proportional to the square of amplitude and square of the frequency (Kaiser 1990). In the discrete time domain, the Teager operator can be defined as:

$$
\Phi[n]=x[n]^{2}-x[n+1] x[n-1] .
$$

TE is obtained by applying the moving average Teager energy over an $\mathrm{M}$ samples window and after which the square root of its absolute value is taken.

$$
\mathrm{TE}(\mathrm{n})=\left|\frac{1}{\mathrm{M}} \sum_{\mathrm{m}=-\frac{\mathrm{M}}{2}}^{+\frac{\mathrm{M}}{2}} \Phi\left[\mathrm{x}_{\mathrm{f}}[\mathrm{m}+\mathrm{n}]\right]\right|^{\frac{1}{2}},
$$

where $\mathrm{M}$ is the number of samples of the moving window applied (30 seconds) and $\mathrm{x}[\mathrm{n}]$ is the EHG signal. This window length and bandwidth were chosen on the basis of the results of a previous study (Benalcazar-Parra et al 2017b). TE(n) was then downsampled to $4 \mathrm{~Hz}$ to match the IUP sampling frequency.

As mechanical activity is a direct consequence of myoelectrical activity, the IUP signal is delayed in regard to the EHG signal, and was adjusted by the cross-correlation function (CCF) in (3). The maximum CCF value gives the time lag that must be adjusted to synchronize TE and IUP signals before obtaining linear regression models. 


$$
C C F[i]=\frac{R_{x y}[i]-\bar{x}[n] \cdot \bar{y}[n]}{\sqrt{\left(R_{x x}[0]-\bar{x}[n]^{2}\right)\left(R_{y y}[0]-\bar{y}[n]^{2}\right)}} \forall i \in[-N, N]
$$

where $\mathrm{x}[\mathrm{n}]$ is the TE signal and $\mathrm{y}[\mathrm{n}]$ the IUP signal.

In addition to estimating the IUP signal itself based on the EHG TE, we also proposed estimating other UA parameters of clinical interest, such as the peak amplitude of each contraction and the tone. A contraction was registered if a significant rise in amplitude $(>20 \mathrm{mmHg})$ was present in the IUP record with a minimum duration of $30 \mathrm{~s}$. The peak amplitude series $\mathrm{A}_{\mathrm{IUP}}(\mathrm{i})$ and $\mathrm{A}_{\mathrm{TE}}(\mathrm{i})$ are formed from the maximum values of the IUP record and TE, respectively, during each contraction (i). Basal series (Basal ${ }_{\mathrm{IUP}}$ and $\mathrm{Basal}_{\mathrm{TE}}$ ) contain the basal tone values computed as the fifth percentile in the 5 minutes prior to the contraction peak amplitude. In this way we have the same number of observations in basal and peak amplitude series for each record. As a result of this process, a total of 3 pairs of series (IUP-TE, $\mathrm{A}_{\mathrm{IUP}}-\mathrm{A}_{\mathrm{TE}}$, Basal $\mathrm{IUP}_{\mathrm{IU}}-\mathrm{Basal}_{\mathrm{TE}}$ ) are available for the design of the different estimation models (see Figure 2).

\subsection{Linear Regression models}

We performed individual, global and adaptive estimates using linear regression for each pair of series. A linear regression can be denoted as:

$$
\hat{Y}=\mathrm{bX}+\mathrm{c}
$$

For each model we then calculate the coefficients of a linear regression model using the least squares method, which yields the following expressions to obtain the coefficients:

$$
\begin{gathered}
b=\frac{s_{X Y}}{S_{X}^{2}}, \\
\mathrm{c}=\bar{y}-\mathrm{b} \bar{x} .
\end{gathered}
$$

Where $S_{X Y}$ is the covariance between $\mathrm{X}$ and $\mathrm{Y}, S_{X}^{2}$ is the variance of $\mathrm{X}, \bar{y}$ is the mean value of $\mathrm{Y}$ and $\bar{X}$ is the mean value of $X$.

Individual models were built using the pairs of series from each woman ( $k$, for $k=1$ to 22$)$ to obtain individual coefficients $b_{k}$ and $c_{k}$ which are then applied to the $\mathrm{TE}_{\mathrm{k}}$ to obtain the estimated $\mathrm{IUP}_{\mathrm{k}}$ for each volunteer. Global models are obtained by concatenating each pair of series of all the patients except one ( $k$, for $k=1$ to 22), for validation purposes (leave-one-out method). The global coefficients $b_{k}$ and $c_{k}$ from the linear regression are then calculated and applied to the $\mathrm{TE}_{\mathrm{k}}$ of the patient left out of the model to obtain its estimated $\mathrm{IUP}_{\mathrm{k}}$. Finally, an adaptive algorithm (see Figure 3 ) is applied to the estimated IUP feature from the global model to fine tune the estimation coefficients for the woman being monitored and deal with inter-patient variability. 
The algorithm consists of applying a number of rules based on clinical knowledge of the IUP signal. In the active phase of labor, contractions can reach a maximum pressures of between $50-80 \mathrm{mmHg}$ (Gilbert 2011) or even greater when oxytocin or prostaglandins are used to induce labor, and the basal tone can have values of $16 \pm 7 \mathrm{mmHg}$ (Dowdle 2003). We therefore set peak amplitude and tonus threshold levels $\left(\right.$ Contr $_{\min }$, Contr $_{\max }$; Tonus $\mathrm{max}_{\max }$, Tonus ${ }_{\min }$ ) to coincide with the expected range of the maximum contraction amplitude and tonus of the IUP global estimation. The following iterative algorithm is applied to 10-minute windows of the IUP estimate. First the tonus rule is checked. Percentile 5 (P5) of the IUP estimate must fall between Tonus $\min _{\text {an }}$ and Tonus max $_{\text {ox }}$ otherwise coefficient $\mathrm{c}$ is updated to set P5 to the mid tonus range $\left(\left(\right.\right.$ Tonus $_{\min }+$ Tonus $\left.\left._{\max } /\right) / 2\right)$. The peak amplitude rule is then checked, i.e. the $95^{\text {th }}$ percentile (P95) must fall between Contr ${ }_{\min }$ and Contr $_{\max }$ otherwise coefficient $b$ is updated to set P95 to the mid contraction peak range $\left(\left(\right.\right.$ Contr $\left.\left._{\min }+\operatorname{Contr}_{\max }\right) / 2\right)$. Then P5 is again checked to be within tonus range, the loop is finished if fulfilled, or re-entered to adjust coefficients otherwise. Initial values of $\mathrm{b}$ and $\mathrm{c}$ are those obtained from the global model. Rules were checked every 10 min and $\mathrm{b}$ and $\mathrm{c}$ were updated if necessary and then used in the current and subsequent 10 -min window.

In order to determine the combination of thresholds that gave rise to the minimum IUP estimate error (optimal combination), the adaptive algorithm was applied with different combinations of the Contr ${ }_{\text {min }}$

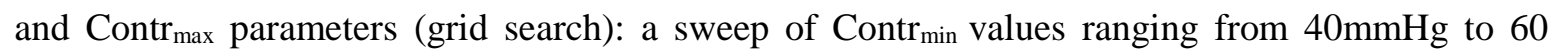
$\mathrm{mmHg}$, and Contr max $_{\text {from }} 80 \mathrm{mmHg}$ to $90 \mathrm{mmHg}$ with a step increment of $5 \mathrm{mmHg}$ in both cases. In the grid search for the optimal selection of thresholds of the adaptive model, the error obtained in the estimation of the concatenated series of the 21-women (training dataset) was evaluated for each global model. The threshold values with the lowest average error on the training dataset were selected. These 'optimal thresholds' were then applied to all the leave-one-out validation patients and the 3 types of errors were obtained for the adaptive estimation. Tonus $\min _{\text {in }}$ and Tonus ${ }_{\max }$ were set to $10-25 \mathrm{mmHg}$. Other values were tested with no significant influence on the results.

Moreover, in contrast to previous studies, three different optimizing signal features were used in the linear regression: continuous pressure, maximum pressure and tonus. Three different models were built for each optimizing signal feature: individual, global and adaptive models, giving a total of 9 models (see Table 2). Continuous pressure models were built using the IUP and TE series as Y and $\mathrm{X}$ in the linear regression, while maximum pressure models and tonus pressure models used the $\mathrm{A}_{\mathrm{IUP}}$, $\mathrm{A}_{\mathrm{TE}}$ and Basal $\mathrm{IUP}_{\mathrm{I}}, \mathrm{Basal}_{\mathrm{TE}}$ series, respectively. The following rules and thresholds were applied to the adaptive models according to the grid search results: values of Contr ${ }_{\min }$ and Contr max $_{\max }$ were set to 45 and $80 \mathrm{mmHg}$, respectively, for the adaptive continuous pressure model (CP_A). A grid search was not performed for the adaptive maximum pressure model (MP_A) and the maximum amplitude rule 
was to maintain the P95 from the original MP_G model (since this model was optimized to yield the best maximum amplitude estimations). Contr ${ }_{\min }$ and Contr $_{\max }$ values were set to 55 and $80 \mathrm{mmHg}$ for the tonus adaptive model (T_A), according to the optimal combination from the grid search results, while the tonus rule kept the P5 from the original T_G model (since the T_G model was optimized to yield the best tonus estimation).

\subsection{Error measures}

The 9 models were evaluated computing the root mean square error of the three estimates: continuous pressure, maximum pressure and tonus

$$
R M S e=\sqrt{\frac{\sum_{i=1}^{N}\left(y_{i}-\hat{y}_{i}\right)^{2}}{N}}
$$

For continuous pressure error $(\mathrm{CPe}), y$ and $\hat{y}$ were the IUP record and the estimated IUP, respectively. For maximum pressure error (MPe), $y$ and $\hat{y}$ were the series containing the maximum pressure of each contraction in the IUP recording and in the estimated IUP, respectively. For the tonus error (TOe), $y$ and $\hat{y}$ were the series of the 5-minutes tonus before the peak of each contraction in the IUP records and in the estimated IUP. These 3 errors were computed for each of the three optimizing signal features (continuous pressure, maximum pressure and tonus) and the three subtypes of estimators (individual, global and adaptive) of the models.

For each optimization signal feature and considering its associated error, the individual model would be the best possible linear estimation of constant coefficients in each case, but as it requires a simultaneous IUP signal to obtain the coefficients of the model it therefore would not be practical in clinical practice. Its results can thus be considered as a reference for the global and adaptive approaches, which do not require an IUP recording in the testing sessions. The reference method for each type of error was therefore established as follows: CP_I for CPe, MP_I for the MPe and T_I for TOe (shown by an arrow in Figure 6). For each error, all the IUP estimation methods were compared with the reference by the Dunnet test to determine whether there were any significant statistical differences $(\alpha=0.05)$.

\section{Results}

Figure 4 shows the simultaneous IUP, TOCO and bipolar EHG recordings and the corresponding EHG Teager parameter (TE). It can be seen that the IUP signal morphology is more reliably reproduced by the TE signal than by the TOCO recording.

Figure 5 shows the IUP estimations of a patient obtained by the 3 different optimizing signal features: continuous pressure (a), maximum pressure (b) and tonus (c); and the three individual, global and 
adaptive approaches. It can be seen that all the methods reproduce well the onset and end of the contractions recorded in the IUP. For continuous pressure estimations (a), the individual model fits best the IUP recording. The adaptive model yields an estimate closer to IUP than that of the global model, especially in the basal periods. For maximum pressure estimates (b), the individual and global models are close to the IUP contraction peak amplitudes, but clearly fail in the non-contractile periods. The adaptive algorithm provides good IUP estimates for almost the entire recording, as it does for the tonus estimations (c). The individual and global models match well with the IUP tonus, but the amplitudes in contractile periods do not.

Table 3 shows the coefficients of variation of linear regression coefficients $b$ and $c$ from the individual and global models. In the former case, the coefficients of variation of slope (b) are high, ranging from $88 \%$ to $146 \%$ and those of intercept (c) range from $38 \%$ to $81 \%$. Lower coefficients of variation are obtained for the global models.

Table 4 shows the mean and standard deviation of the different types of errors measured in RMSe for each IUP estimation method: continuous pressure error (CPe), maximum pressure error (MPe) and tonus error (TOe). Mean values are also shown in the form of bar plots in Figure 6 for ease of comparison. The statistically significant differences with the reference methods (indicated by an arrow) are also shown in Figure 6 by an asterisk.

It can be seen that the adaptive model outperformed the global model in almost all cases (grey bars lower than orange bars). For continuous pressure errors (CPe) the mean value of the CP_I model was $9.62 \mathrm{mmHg}$, significantly lower than the global model $(14.61 \mathrm{mmHg})$, but not significantly lower than any of the adaptive models, with mean values around $12 \mathrm{mmHg}$. On the other hand, the CPe of the individual and global models with the other optimizing signal features were significantly higher with mean values over $17 \mathrm{mmHg}$ for the tonus optimizing models, and higher than $30 \mathrm{mmHg}$ for those optimizing maximum pressure.

As regards the maximum pressure error, it can be seen that MPe almost doubles CPe for continuous pressure estimations). A mean error of about $30 \mathrm{mmHg}$ is obtained for the $\mathrm{CP} \_\mathrm{G}$ model. The models designed for maximum pressure estimations provide the best results and reduce this error to about $15 \mathrm{mmHg}$ for the global (MP_G) and adaptive (MP_A) models. The individual and global models derived from tonus optimization yielded the highest error by far in estimating maximum pressure values (MPe were greater than $38 \mathrm{mmHg}$ ) and the adaptive model significantly improved this error $(18.02 \pm 6.66 \mathrm{mmHg})$. When comparing all the methods with the reference model (MP_I), they all had statistically significantly higher values, except for MP_G and MP_A.

The models optimized for continuous pressure are seen to provide very low tonus errors $(3.2 \mathrm{mmHg}$, 7.80mmHg and 5.08mmHg for CP_I, CP_G and CP_A respectively), similar to those obtained from 
those optimized for tonus estimation $(2.26 \mathrm{mmHg}, 5.18 \mathrm{mmHg}$ and $5.61 \mathrm{mmHg}$ for T_I, T_G and T_A respectively). Those optimized for maximum pressure show errors greater than $35 \mathrm{mmHg}$ in tonus estimation (TOe>), except for the adaptive approach (mean Toe $=6.08 \mathrm{mmHg}$ ). No statistically significant difference was found in any of the adaptive models as compared to the reference model (T_I).

Simultaneous TOCO recordings were also available in a database subset ( $\mathrm{N}=7$ women) for additional comparison with the most commonly used non-invasive technique in clinical settings. Table 5 shows the mean and standard deviation of the different types of error in the IUP estimations for the TOCO and EHG-based adaptive models. The results of the rest of the models are not shown for the sake of clarity. Statistically significant lower error values $(\mathrm{p}<0.05)$ than the TOCO values were obtained for all the adaptive models: around $10 \mathrm{mmHg}$ lower in the $\mathrm{CPe}, 11 \mathrm{mmHg}$ in the $\mathrm{MPe}$ and $8 \mathrm{mmHg}$ in the TOe. The better performance of the adaptive estimations over TOCO can also be seen in Figure 7, where the IUP estimation with CP_A matches better with the IUP recording than the TOCO, and also gives a better amplitude and basal tone estimates.

\section{Discussion}

Monitoring UA is important during pregnancy and labor; accurate information on uterine dynamics is essential since a rise in UA during the first and second labor stages may increase the risk of adverse fetal outcomes (Bakker et al 2007). Contraction duration, frequency, tone and intensity are important parameters for fetal well-being. Although measuring intrauterine pressure by pressure catheter is the only reliable method of obtaining precise UA information, this invasive technique is only applied after the membranes have broken and is not recommended for long-term monitoring. At the present time, the only non-invasive method of monitoring uterine dynamic is by tocodynamometry. Since this technique uses a strain gauge transducer to indirectly estimate IUP by measuring the changes in abdominal contours, it can only reliably obtain the number of contractions. Statistically significant differences in contraction amplitudes between TOCO and IUP have been reported in the literature from twenty patients at between 36 and 41 weeks' gestation during active labor (TOCO: $29 \mathrm{mmHg}$ vs IUP: $44.6 \mathrm{mmHg}$; $\mathrm{p}=0.013$ with a correlation coefficient $\mathrm{r}=0.26$ ) (Miles et al 2001). The present results also show the unreliability of this technique, with error values of $21.93 \mathrm{mmHg}$ in the TOCO recording, $26.97 \mathrm{mmHg}$ in maximum contraction amplitude and $13.96 \mathrm{mmHg}$ in basal tone.

We here propose a non-invasive method of estimating IUP based on linear regression models and the Teager method proposed by (Rooijakkers et al 2014) to assess the energy in the EHG signal. Estimating the IUP signal by calculating the Teager operator has been reported to obtain better results than computing the RMS and UNFM (Benalcazar-Parra et al 2017b, Rooijakkers et al 2014). 
Different IUP estimation models were built using different optimizing signal features. Individual models were then designed from individual patient datasets. These individual models, although not clinically applicable due to not being generalizable, provide a reference for the minimum error obtainable from a linear model using TE from a single EHG channel. These models reflected the wide variations among the patients in the form of high coefficient variability.

Global models were designed following a leave-one-out validation method to assess how the model accuracy generalizes to an independent patient dataset. The coefficient variability was largely reduced, only the slope of tonus global model still showed high CV due to the small absolute values of such slope, and the smaller influence of it in comparison to the intercept. Our continuous pressure global model showed similar results to those obtained by (Rooijakkers et al 2014) (RMS error:14.6 $\mathrm{mmHg}$ vs $13.9 \mathrm{mmHg})$. The slight difference $(0.7 \mathrm{mmHg})$ in the results can be explained by the following factors: the number of subjects involved (present study: $\mathrm{N}=22$ vs Rooijakers: $\mathrm{N}=7$ ) and different electrode positions. The signal processing system used in the present work was also different from that in (Rooijakkers et al 2014); ours was based on the group's previous results focused on optimizing the bandwidth and window length for IUP estimations by computing the Teager operator (Benalcazar-Parra et al 2017b). Neither did we remove the baseline tone, which is important when estimating certain conditions such as hypertonic or hypotonic uterine dysfunctions (Cunningham et al 2010).

Inter-patient variability is one of the main limitations of a global IUP estimation model. Differences in body constitution or obstetrical factors are responsible for these variations that mainly affect amplitude. Other factors that can also contribute to it are: skin preparation, electrode quality and different physiological factors, such as temperature, metabolic and ionic changes, which can affect myocyte cellular processes (Gamet et al 1996) thus affecting the EHG signal. (Skowronski et al 2006) proposed a method of reducing inter-patient variability by means of a causal FIR Wiener filter and obtained a good resemblance to the IUP signal. However, as an initialization period of 10 minutes of IUP recording is required it is impractical for clinical monitoring. In contrast, in the present work, adaptive models were developed to deal with the inter-patient variability problem and to improve the estimated IUP accuracy by tuning and updating the coefficients of the global models for each subject. The adaptive version of the continuous pressure model reduced the IUP signal estimation error and, unlike the global model, any differences with the reference (individual) model were not significant. The adaptive models were based on prior knowledge of the characteristics of the IUP signal and simple rules were established regarding the values of the peak amplitude and tone of the IUP signal within the physiological ranges of the active phase of labor. This type of approach means that the adaptive models are not only generalizable, since they are from the global model with the leave-one- 
out approach, but also reduce inter-patient variability and avoid unrealistic IUP estimate values, e.g. negative or above $100 \mathrm{mmHg}$. Nonetheless, as the threshold values used for tuning the model coefficients were set to monitor the active labor period, they may need to be adjusted for use in other conditions, e.g. during regular pregnancy check-ups. On the other hand, the amplitude and tone rules were verified iteratively with the calculation of the 95th and 5th percentile, respectively, of the global estimate in a ten-minute analysis window. This provides some robustness at abnormally high values of TE EHG for possible artifacts or flat-lines, but the coefficients (and results of the IUP estimate) could be affected until the next analysis window if they occur over a long period. We also assessed multivariate models with additional obstetrical information (results not shown), but they did not improve on our adaptive approach, probably due to the small database.

Previously reported methods of estimating IUP (Rooijakkers et al 2014, Rabotti et al 2008, Benalcazar-Parra et al 2017b) focused on minimizing the mean square error of the signal estimates (continuous pressure optimization) and did not assess the error in estimating clinically useful parameters, such as maximum contractile pressure and basal tone. These are relevant indicators of labor progress and maternal-fetal well-being and of conditions such as tachysystole, hyperstimulation or hypertonus. In general, the individual, global and adaptive continuous pressure models give good estimates of the entire IUP signal and tonus, but not of peak contraction amplitude. On the other hand, the global models described here, which specifically optimize maximum pressure and tonus estimations, provided satisfactory clinically relevant parameters, but failed to estimate the whole IUP signal. To improve the CPe of the global models, we also developed an adaptive version of these maximum pressure and tonus estimators. The proposed adaptive models outperformed the global models (of constant coefficients) and yielded the best balance between the errors of the different optimizing signal features. Furthermore, direct comparison of these EHG-based estimators with tocodynamometer measurements showed that they clearly outperform the traditional TOCO recordings. The proposed adaptive models are therefore suitable for use in clinical settings for noninvasive estimation of the IUP signal, contraction peak pressure and tonus.

Despite the clear advantages of our proposed IUP signal feature estimates they are not exempt from limitations. First, the global models use linear regression models to reproduce a non-linear physiological process. In this regard, other non-linear regression techniques such as neural networks could be explored to improve the estimated intrauterine pressure. Secondly, the delay between mechanical and electrical activity depends on many aspects, it is especially dependent on the EHG propagation pattern, which may vary even between contractions (Devedeux et al 1993). For the sake of simplicity, we computed the CCF between the whole recording segments of TE and IUP signal before obtaining model coefficients of the linear regression in order to adjust this delay. A more 
precise adjustment of this delay in each contraction may provide slightly better estimation than those reported in this work, even though this would complicate the method and require individually segmented contractions and therefore would be clinically limited. The EHG-based IUP estimation would always be ahead of the real IUP (since the myoelectrical activity precedes the resulting mechanical activity). However, this small time lag would not significantly affect clinical monitoring and interpretation. Thirdly, the estimation was based on only one EHG characteristic (Teager energy), which mainly evaluates the intensity of ongoing myoelectrical activity, while multivariable models could enrich the estimate. Fourthly, we used local information sensed by two electrodes for the IUP estimates, whereas intrauterine pressure is a consequence of all the events in the entire uterus. Multichannel estimations could thus also be made prior to applying the adaptive algorithm. However, low quality signals from some electrodes could give even worse estimates of intrauterine pressure, as in the case of the multichannel model proposed in (Rooijakkers et al 2014), where the horizontal channel (L1) yielded an RMSe value of $13.9 \mathrm{mmHg}$, while the vertical channel (L2) gave a higher value $(20.4 \mathrm{mmHg})$. Finally, since there is no IUP recording available in the non-active labor period, our approach was only validated for the active labor period.

\section{Conclusions}

In the present study several models based on linear regression were used to make IUP estimations from the EHG signal. Inter-patient variability was mitigated by an adaptive algorithm to tune the estimation coefficients and improve accuracy. The results obtained show that continuous pressure estimation by a global model accurately estimates the IUP signal and tone, but provides a poor estimate of maximum contraction pressure, which is an important factor in clinical practice. The proposed estimation model for maximum pressure can mitigate this limitation. The proposed adaptive models improved continuous pressure estimation and provided better balanced estimates of the IUP signal, tone and peak pressure than the global models with constant coefficients and clinically noninvasive relevant information. They also significantly improved IUP estimation in comparison to the traditional TOCO recordings. The proposed EHG-based IUP estimation models achieved more accurate monitoring of uterine activity and therefore a better assessment of labor progress and maternal and fetal well-being.

\section{Funding}

This research project was supported by the Spanish Ministry of Economy and Competitiveness, the European Regional Development Fund (DPI2015-68397-R), and by the projectsUPV_FE-2018C03 and GV/2018/104. 


\section{References}

Alberola-Rubio J, Garcia-Casado J, Prats-Boluda G, Ye-Lin Y, Desantes D, Valero J and Perales A 2017 Prediction of labor onset type: Spontaneous vs induced; role of electrohysterography? Comput. Methods Programs Biomed.144 127-33

Alberola-Rubio J, Prats-Boluda G, Ye-Lin Y, Valero J, Perales A and Garcia-Casado J 2013a Comparison of non-invasive electrohysterographic recording techniques for monitoring uterine dynamics Med. Eng. Phys.35 1736-43

Alberola-Rubio J, Prats-Boluda G, Ye-Lin Y, Valero J, Perales A and Garcia-Casado J 2013b Comparison of non-invasive electrohysterographic recording techniques for monitoring uterine dynamics Med. Eng. Phys.35 1736-43

Bakker P C A M, Van Rijsiwijk S, van Geijn H P and van Geijn H P 2007 Uterine activity monitoring during labor J. Perinat. Med.35 468-77

Benalcazar-Parra C, Montfort-Orti R, Ye-Lin Y, Alberola-Rubio J, Perales Marin A, Mas-Cabo J, Garcia-Casado J and Prats-Boluda G 2017a Characterization of Uterine Response to Misoprostol based on Electrohysterogram Proceedings of the 10th International Joint Conference on Biomedical Engineering Systems and Technologies (SCITEPRESS - Science and Technology Publications) pp 64-9

Benalcazar-Parra C, Sempere C and Marin A P 2017b Improvement of non-invasive intrauterine pressure estimation based on Electrohysterogram XXXV Congreso Anual de la Sociedad Española de Ingenieréa Biomédica (Bilbao) pp 225-8

Caldeyro-Barcia R, Sica-Blanco Y, Poseiro J J, González Panizza V, Méndez-Bauer C, Fielitz C, Alvarez H, Pose S V. and Hendricks C H 1957 A quantitative study of the action of synthetic oxytocin on the pregnant human uterus J. Pharmacol. Exp. Ther.121 18-31

Cunningham F G, Gant N F, Leveno K J, Gilstrap L C, Hauth J C and Wenstrom K D 2010 Williams Obstetrics (McGraw-Hill Professional)

Devedeux D, Marque C, Mansour S, Germain G and Duchêne J 1993 Uterine electromyography: a critical review. Am. J. Obstet. Gynecol.169 1636-53

Dowdle M A 2003 Comparison of two intrauterine pressure catheters during labor. J. Reprod. Med.48 501-5

Euliano T Y, Nguyen M T, Darmanjian S, McGorray S P, Euliano N, Onkala A and Gregg A R 2013 Monitoring uterine activity during labor: a comparison of 3 methods Am. J. Obstet. Gynecol.20866.e1-66.e6

Fergus P, Cheung P, Hussain A, Al-Jumeily D, Dobbins C and Iram S 2013 Prediction of Preterm Deliveries from EHG Signals Using Machine Learning PLoS One8

Freeman R K, Garite T J, Nageotte M P and Miller L A 2012 Fetal heart rate monitoring (Philadelfia: Lippincott Williams \& Wilkins)

Gamet D, Duchêne J and Goubel F 1996 Reproducibility of kinetics of electromyogram spectrum parameters during dynamic exercise Eur. J. Appl. Physiol. Occup. Physiol.74 504-10

Gilbert E S 2011 Manual of high risk pregnancy \& delivery (Mosby Elsevier) 
Harper L M, Shanks A L, Tuuli M G, Roehl K A and Cahill A G 2013 The risks and benefits of internal monitors in laboring patients Am. J. Obstet. Gynecol.20938.e1-38.e6

Hassan M M, Terrien J, Muszynski C, Alexandersson A, Marque C and Karlsson B 2013 Better Pregnancy Monitoring Using Nonlinear Correlation Analysis of External Uterine Electromyography IEEE Trans. Biomed. Eng.60 1160-6

Hassan M, Terrien J, Alexandersson A, Marque C and Karlsson B 2010 Improving the classification rate of labor vs. normal pregnancy contractions by using EHG multichannel recordings 2010 Annual International Conference of the IEEE Engineering in Medicine and Biology (IEEE) pp 4642-5

Jezewski J, Horoba K, Matonia A and Wrobel J 2005 Quantitative analysis of contraction patterns in electrical activity signal of pregnant uterus as an alternative to mechanical approach Physiol. Meas.26 753-67

Kaiser J F 1990 On a simple algorithm to calculate the "energy" of a signal International Conference on Acoustics, Speech, and Signal Processing (IEEE) pp 381-4

Lucovnik M, Maner W L, Chambliss L R, Blumrick R, Balducci J, Novak-antolic Z and Garfield R E 2011 Noninvasive Uterine Electromyography For Prediction of Preterm Delivery * Am J Obs. Gynecol3228.e1-228.10

Maner W L, Garfield R E, Maul H, Olson G and Saade G 2003 Predicting term and preterm delivery with transabdominal uterine electromyography Obstet. Gynecol.101 1254-60

Miles A M, Monga M and Richeson K S 2001 Correlation of External and Internal Monitoring of Uterine Activity in a Cohort of Term Patients Am. J. Perinatol.18 137-40

Mischi M, Chen C, Ignatenko T, de Lau H, Ding B, Oei S G G and Rabotti C 2018 Dedicated Entropy Measures for Early Assessment of Pregnancy Progression From Single-Channel Electrohysterography IEEE Trans. Biomed. Eng.65 875-84

Rabotti C, Mischi M, van Laar J O E H, Oei G S and Bergmans J W M 2008 Estimation of internal uterine pressure by joint amplitude and frequency analysis of electrohysterographic signals Physiol. Meas.29 829-41

Rooijakkers M, Rabotti C, Guid Oei S, Aarts R M and Mischi M 2014 Low-complexity intrauterine pressure estimation using the Teager energy operator on electrohysterographic recordings Physiol. Meas. Phys. Eng. Med. Physiol. Meas. Physiol. Meas35 1215-28

Schlembach D, Maner W L, Garfield R E and Maul H 2009 Monitoring the progress of pregnancy and labor using electromyography Eur. J. Obstet. Gynecol. Reprod. Biol.144 S33-9

Skowronski M D, Harris J G, Marossero D E, Edwards R K and Euliano T Y 2006 Prediction of Intrauterine Pressure From Electrohysterography Using Optimal Linear Filtering IEEE Trans. Biomed. Eng.53 1983-9

Yang Z, Yang R and Lu Y 2017 Estimation of Intrauterine Pressure orom Electrohysterography using Hilbert Phase Slips and Statistics Method J. Mech. Med. Biol.17 1750089

Ye-Lin Y, Prats-Boluda G, Alberola-Rubio J, Bueno Barrachina J-M, Perales A and Garcia-Casado J 2013 Prediction of labor using non-invasive laplacian EHG recordings 2013 35th Annual International Conference of the IEEE Engineering in Medicine and Biology Society (EMBC) 
vol 2013(IEEE)pp 7428-31 


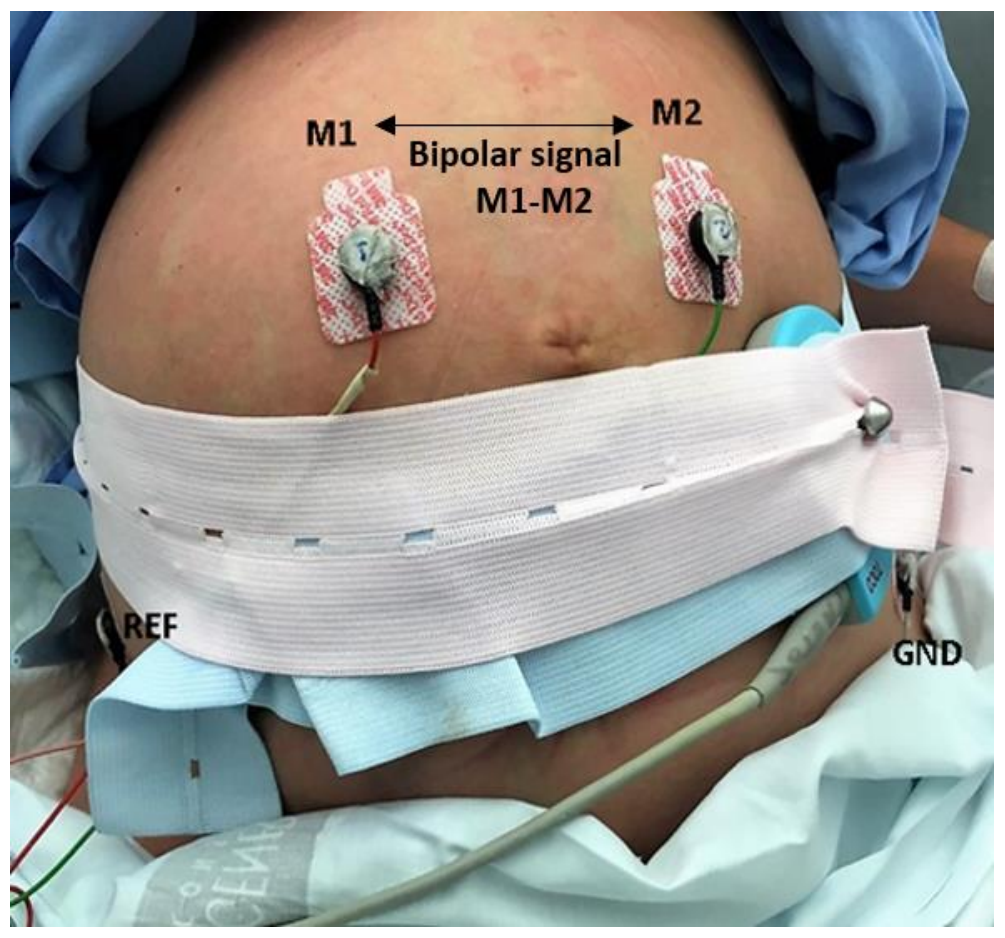

Figure 1. Electrode arrangement for EHG recording.

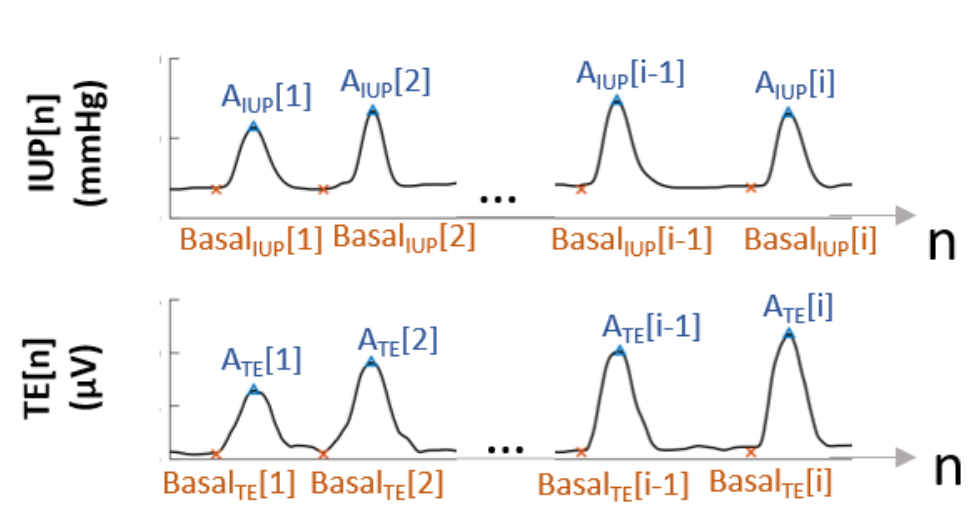

Figure 2. Pairs of series used for linear regression models: IUP(n)-TE(n) for continuous pressure estimation, $A_{I U P}(i)-A_{T E}(i)$ maximum pressure estimation and $\operatorname{Basal}_{I U P}(i)-B \operatorname{Bal}_{\mathrm{TE}}(\mathrm{i})$ for basal estimation. $\mathrm{A}_{\mathrm{IUP}}$ and $\mathrm{A}_{\mathrm{TE}}$ are the peak values of contractions in IUP and TE respectively. Basal IUP and $\mathrm{Basal}_{\mathrm{TE}}$ are from percentile 5 in the 5 minutes prior to peak contraction amplitude. 


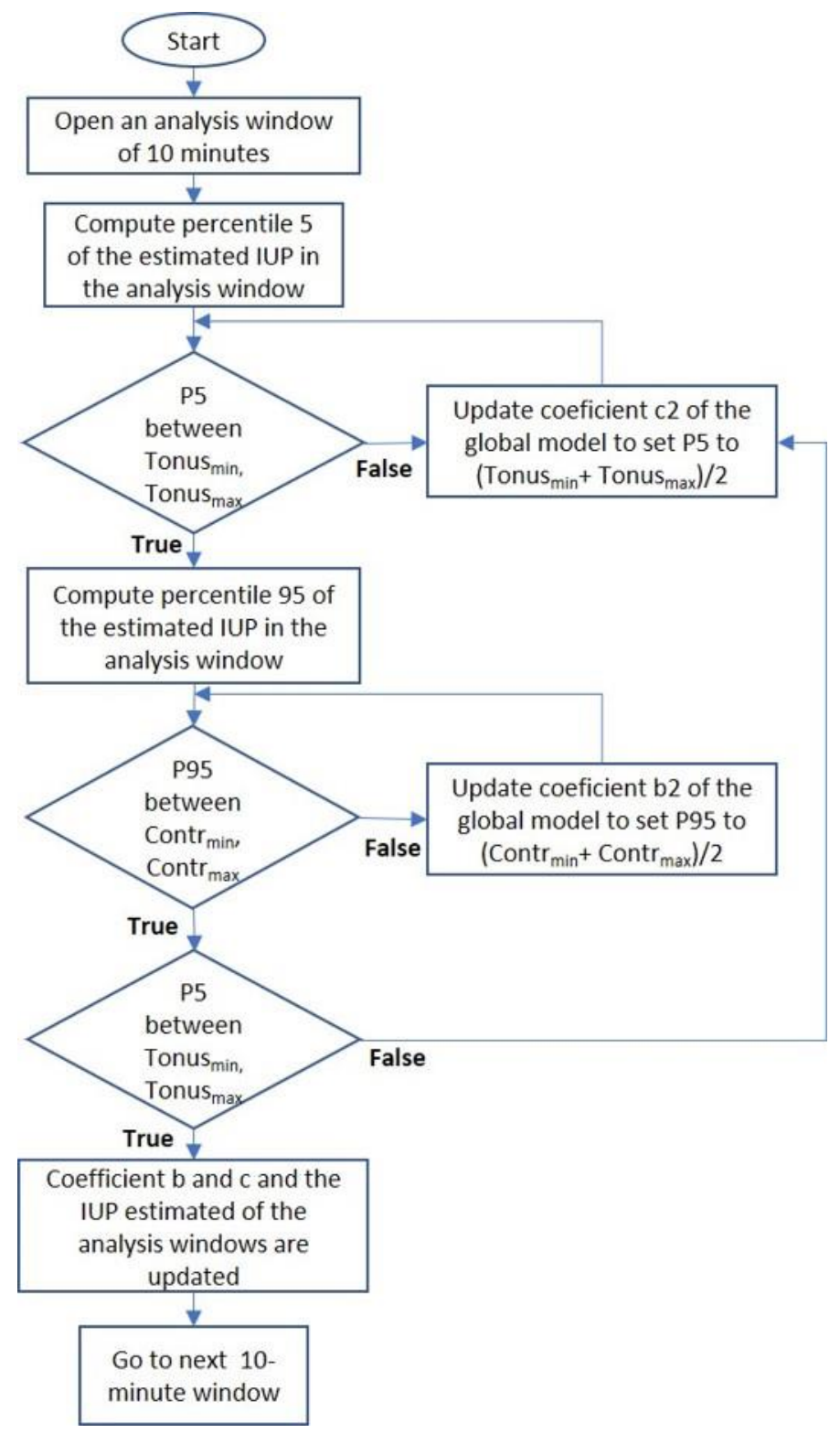

Figure 3. Scheme of the adaptive algorithm proposed for IUP estimation. 

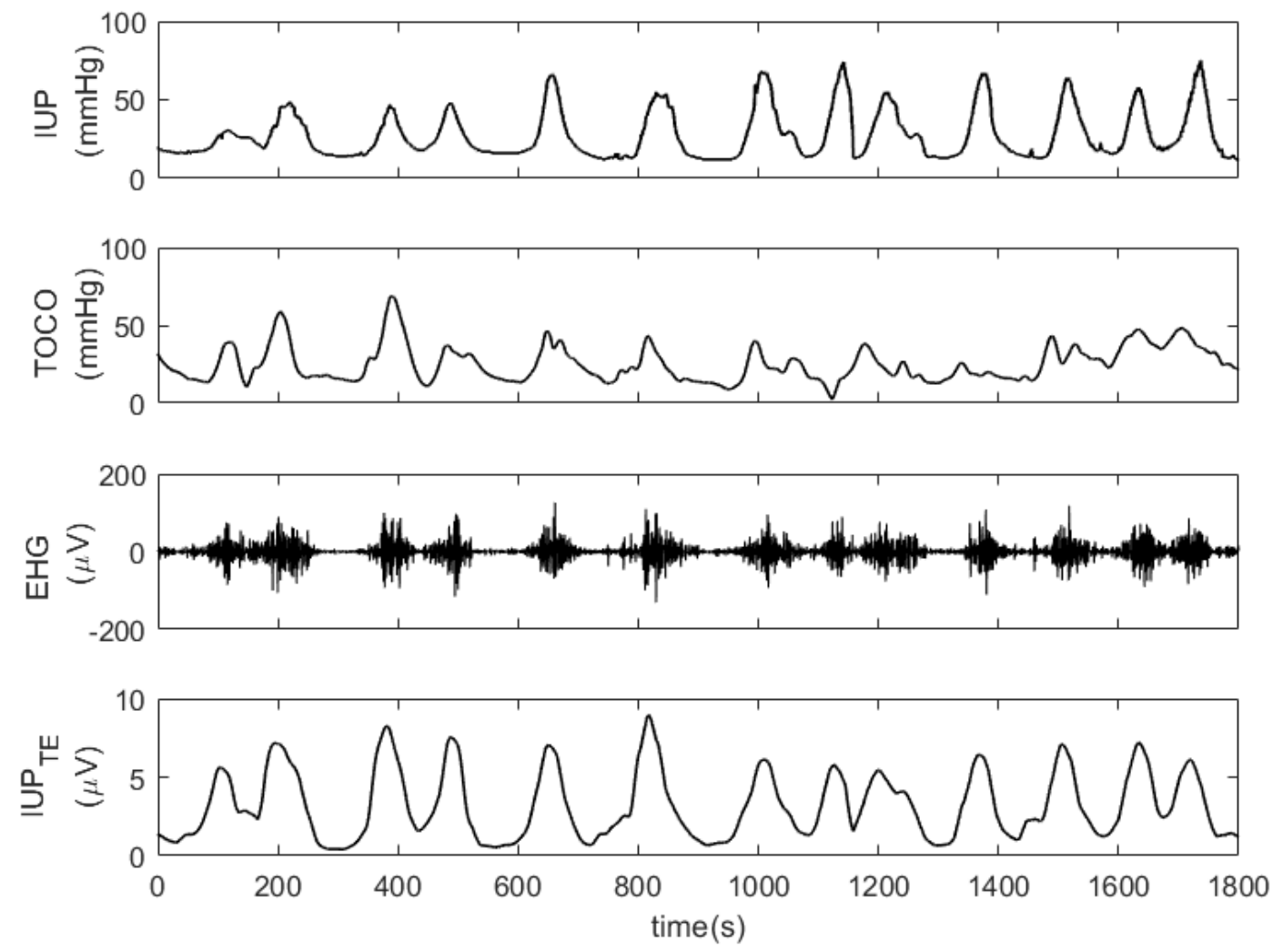

Figure 4. Example of 30 minutes of simultaneous IUP, TOCO, bipolar EHG recordings and Teager parameter computed from the EHG signal. 
(a)
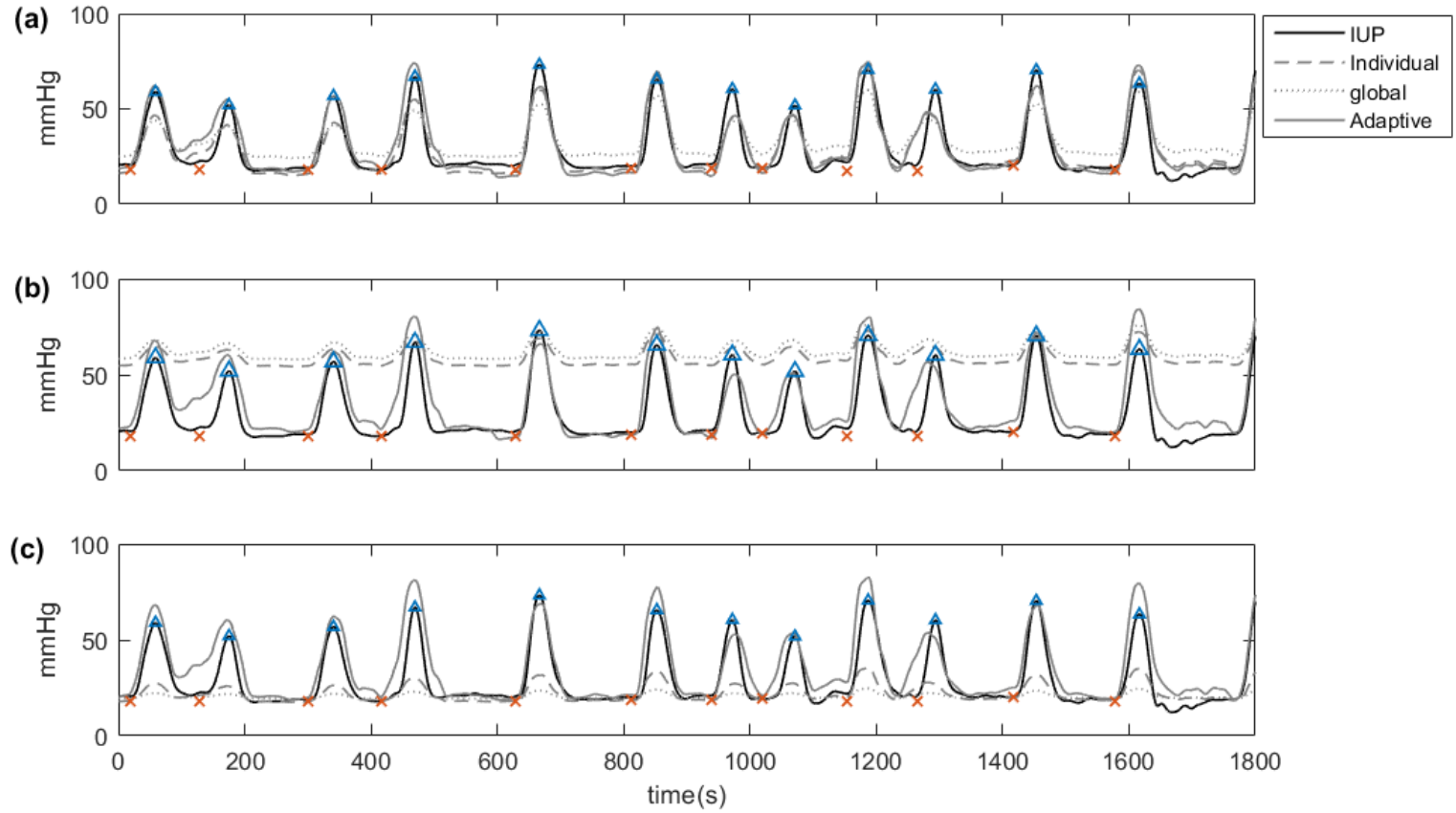

Figure 5. IUP estimates from the different models: (a) continuous pressure models, (b) maximum pressure models and (c) tonus models. Blue triangles indicate maximum contraction peaks and red $\mathrm{x}$ indicates the tonus of the 5 minutes prior to maximum contraction peaks in the IUP recording.

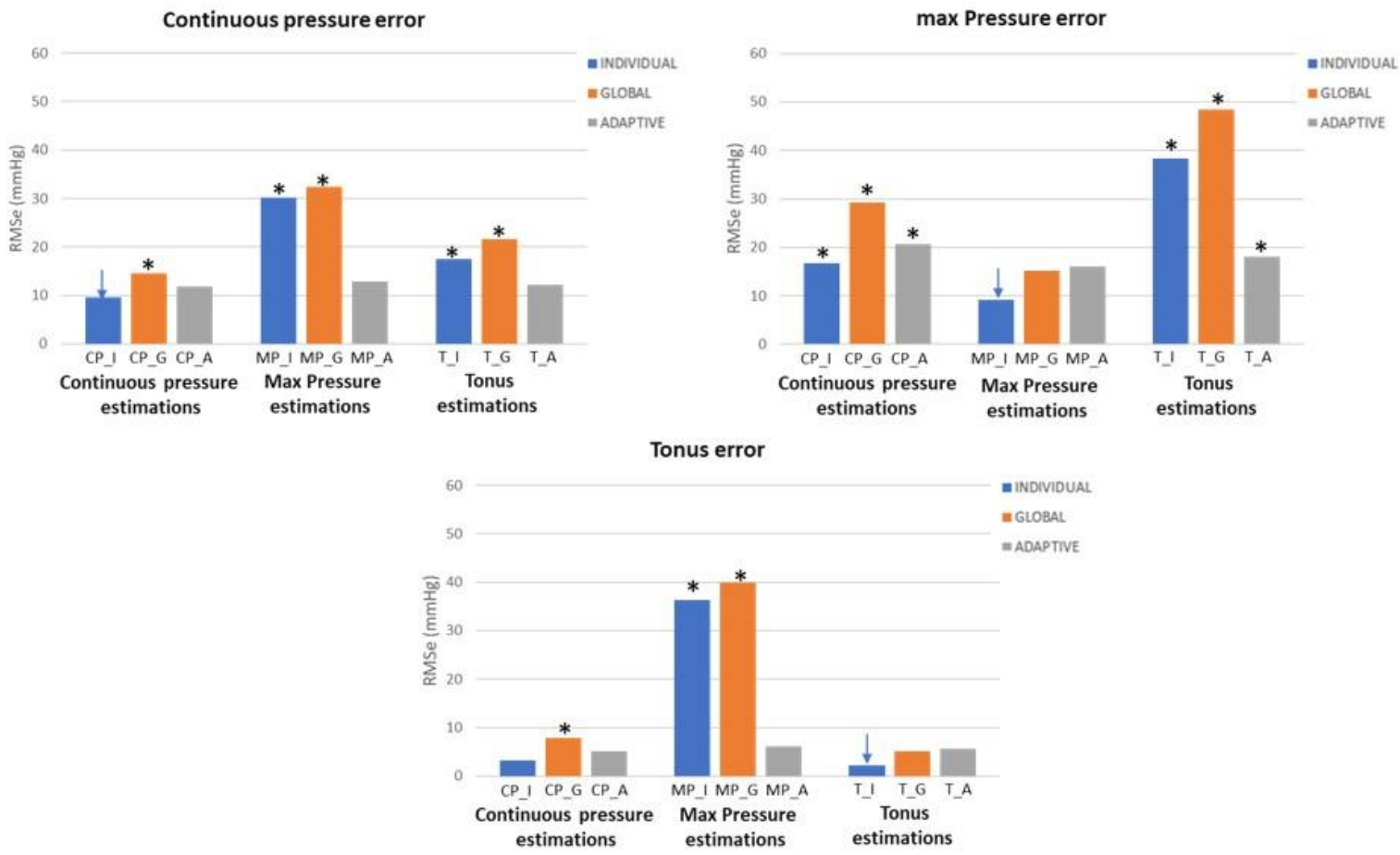

Figure 6. Mean calculated error values (continuous pressure, maximum pressure, and tonus errors) for the different IUP estimates. Statistically significant differences with the reference model (blue arrow) are shown by an asterisk. 


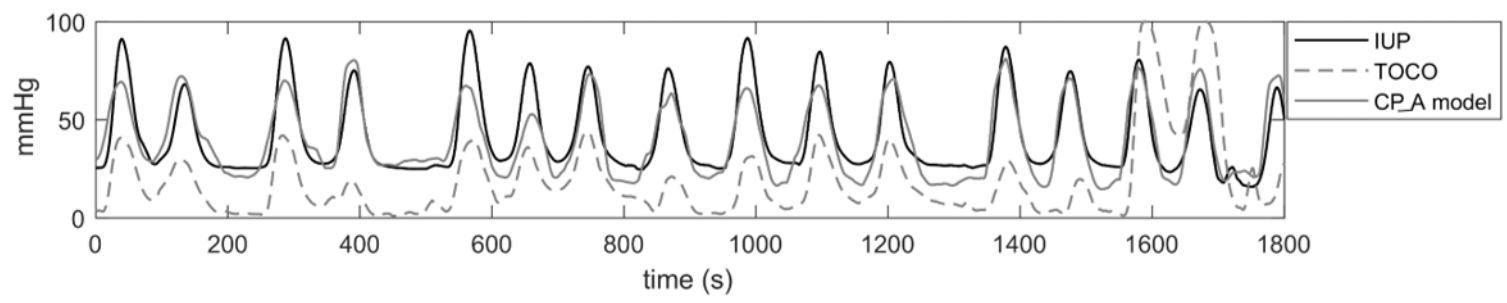

Figure 7. IUP estimation by TOCO and continuous pressure adaptive model from the same patient 
Table 1. Womens' obstetrical characteristics.

\begin{tabular}{|l|c|}
\hline \multicolumn{1}{|c|}{ Variable } & mean \pm std \\
\hline Mother age (years) & $30.80 \pm 5.53$ \\
\hline Gestational age (days) & $280.10 \pm 6.84$ \\
\hline BMI $\left(\mathrm{kg} / \mathrm{m}^{2}\right)$ & $30.16 \pm 3.83$ \\
\hline Gestations & $2.11 \pm 1.30$ \\
\hline Parity & $0.47 \pm 0.82$ \\
\hline Birth weight $(\mathrm{g})$ & $3530.8 \pm 577.8$ \\
\hline
\end{tabular}

Table 2. Models generated for each optimizing signal feature and model type

\begin{tabular}{|l|c|c|c|}
\cline { 2 - 4 } \multicolumn{1}{c|}{} & \multicolumn{3}{c|}{ Model type } \\
\hline $\begin{array}{l}\text { Optimizing signal } \\
\text { feature }\end{array}$ & Individual & Global & Adaptive \\
\hline Continuous pressure & CP_I & CP_G & CP_A \\
\hline Maximum pressure & MP_I & MP_G & MP_A \\
\hline Tonus & T_I & T_G & T_A \\
\hline
\end{tabular}

Table 3. Coefficients of variation of the regression coefficients $b$ and $c$ obtained for individual and global models

\begin{tabular}{|l|r|r|r|r|r|r|}
\cline { 2 - 7 } \multicolumn{1}{c|}{} & CP_I & CP_G & MP_I & MP_G & TO_I & TO_G \\
\hline CV(b) & $88 \%$ & $12 \%$ & $114 \%$ & $8 \%$ & $146 \%$ & $53 \%$ \\
\hline CV(c) & $81 \%$ & $4 \%$ & $38 \%$ & $2 \%$ & $53 \%$ & $1 \%$ \\
\hline
\end{tabular}

Table 4. Mean and standard deviation of different types of error for different types of IUP estimation models.

\begin{tabular}{l|l|c|c|c|}
\cline { 3 - 5 } \multicolumn{2}{c}{} & \multicolumn{3}{c|}{ Error } \\
\cline { 3 - 5 } $\begin{array}{l}\text { Optimizing signal } \\
\text { feature }\end{array}$ & Model Type & CPe $(\mathrm{mmHg})$ & $\mathrm{MPe}(\mathrm{mmHg})$ & Toe $(\mathrm{mmHg})$ \\
\hline \multirow{3}{*}{ Continuous pressure } & Individual & $9.62 \pm 2.22$ & $16.78 \pm 4.75$ & $3.20 \pm 1.49$ \\
\cline { 3 - 5 } & Global & $14.61 \pm 5.60$ & $29.17 \pm 13.09$ & $7.80 \pm 5.15$ \\
\cline { 2 - 5 } & Adaptive & $11.88 \pm 2.67$ & $20.64 \pm 8.26$ & $5.08 \pm 2.76$ \\
\hline \multirow{3}{*}{ Maximum Pressure } & Individual & $30.19 \pm 12.92$ & $9.21 \pm 3.18$ & $36.33 \pm 16.29$ \\
\cline { 2 - 5 } & Global & $32.46 \pm 5.69$ & $15.13 \pm 5.24$ & $39.96 \pm 5.39$ \\
\cline { 2 - 5 } & Adaptive & $12.85 \pm 3.38$ & $16.02 \pm 4.79$ & $6.08 \pm 3.47$ \\
\hline \multirow{3}{*}{ Tonus } & Individual & $17.40 \pm 4.50$ & $38.34 \pm 13.53$ & $2.26 \pm 1.14$ \\
\cline { 2 - 5 } & Global & $21.52 \pm 5.02$ & $48.43 \pm 12.78$ & $5.18 \pm 2.76$ \\
\cline { 2 - 5 } & Adaptive & $12.14 \pm 2.43$ & $18.02 \pm 6.66$ & $5.61 \pm 2.90$ \\
\hline
\end{tabular}


Table 5. Mean and standard deviation of different types of error for adaptive EHG-based IUP estimations and TOCO. ${ }^{*}$ denotes statistically significant difference $(\mathrm{p}<0.05)$ with TOCO error.

\begin{tabular}{|l|c|c|c|}
\cline { 2 - 4 } \multicolumn{1}{c|}{} & \multicolumn{3}{c|}{ Error } \\
\cline { 2 - 4 } \multicolumn{1}{c|}{} & $\mathrm{CPe}(\mathrm{mmHg})$ & $\mathrm{MPe}(\mathrm{mmHg})$ & TOe $(\mathrm{mmHg})$ \\
\hline TOCO & $21.93 \pm 5.89$ & $26.97 \pm 9.16$ & $13.96 \pm 4.83$ \\
\hline CP_A & $10.70 \pm 2.43^{*}$ & $15.91 \pm 4.88^{*}$ & $5.24 \pm 2.91^{*}$ \\
\hline MP_A & $12.78 \pm 3.12^{*}$ & $15.06 \pm 4.16^{*}$ & $6.86 \pm 4.06^{*}$ \\
\hline T_A & $12.20 \pm 3.01^{*}$ & $15.26 \pm 4.20^{*}$ & $5.81 \pm 3.43^{*}$ \\
\hline
\end{tabular}

